Article

\title{
On Factorizable Semihypergroups
}

\author{
Dariush Heidari $^{1}{ }^{\mathbb{D}}$ and Irina Cristea ${ }^{2, * \mathbb{C}}$ \\ 1 Faculty of Science, Mahallat Institute of Higher Education, Mahallat 37811-51958, Iran; \\ dheidari@mahallat.ac.ir or dheidari82@gmail.com \\ 2 Centre for Information Technologies and Applied Mathematics, University of Nova Gorica, \\ Vipavska cesta 13, 5000 Nova Gorica, Slovenia \\ * Correspondence: irina.cristea@ung.si or irinacri@yahoo.co.uk; Tel.: +386-0533-15-395
}

Received: 29 May 2020; Accepted: 17 June 2020; Published: 1 July 2020

check for updates

\begin{abstract}
In this paper, we define and study the concept of the factorizable semihypergroup, i.e., a semihypergroup that can be written as a hyperproduct of two proper sub-semihypergroups. We consider some classes of semihypergroups such as regular semihypergroups, hypergroups, regular hypergroups, and polygroups and investigate their factorization property.
\end{abstract}

Keywords: semihypergroup; factorization; regular semihypergroup; polygroup

\section{Introduction}

The decomposition property of a set appears as a key element in many topics in algebra, being connected, for example, with equivalence relations (and called partition), factorizable semigroups [1,2], factorization of groups [3], breakable semigroups [4], or recently introduced breakable semihypergroups [5]. A group $G$ is called factorized if it can be written as a product of two subgroups $A$ and $B$. This means that any element $g$ in $G$ has the form $g=a b$ for some $a \in A$ and $b \in B$ [3]. One of the most famous results about factorization of groups, proven in 1955 by Ito [6], concerns the product of two abelian subgroups. More precisely, it is proven that any group $G=A B$ written as the product of two abelian subgroups is metabelian. A survey on some topics related to factorization of groups was published in 2009 by Amberg and Kazarin [3]. The problem of the factorization of groups has been immediately extended to the theory of semigroups. We mention here the systematic studies of Tolo [2], Catino [1], or Tirasupa [7]. A semigroup is called factorizable if it can be written as the product of two proper subgroups [1]. Besides the concept of left univocal factorization, $(A, B)$ of a semigroup $S=A B$ is defined by the supplementary condition: for any $a, a^{\prime} \in A$ and $b, b^{\prime} \in B$, the equality $a b=a^{\prime} b^{\prime}$ implies that $a=a^{\prime}$ (and similarly for the right univocal factorization). In the same article [1], after constructing all the semigroups that have a univocal factorization with factors isomorphic with a pair of prescribed semigroups, the author presented necessary and sufficient conditions that a factorization of a semigroup by right simple semigroups be univocal and characterized the semigroups with this factorization property.

Motivated by the above-mentioned studies, in this paper we introduce the notion of the factorizable semihypergroup, and we investigate the factorization property for special semihypergroups, as regular semihypergroups, hypergroups, regular hypergroups, and polygroups. Then, we also present some properties connected with the left (right) univocal factorization. It is worth mentioning here that the problem of decomposition or factorization in hypercompositional algebra has been previously studied by the authors in [5] related with breakable semihypergroups, or by Massouros in [8,9]. In [8], the separation aspect was studied for hypergroups, join spaces, and convexity hypergroups, while in [9], it was proven that from the general decomposition theorems, which are valid in hypergroups, well known decomposition theorems for convex sets were derived as corollaries, like Kakutani's lemma, Stone and Helly's theorem, etc. 


\section{Preliminaries}

For the basic concepts and terminology of semihypergroups or hypergroups, the reader is refereed to the fundamental books [10-12]. In the following, we will recall those related to the identity element, invertible element, or zero element, as well as the basic properties of regular semihypergroups and regular hypergroups, or polygroups. See also $[13,14]$ for more details regarding small polygroups.

Let $S$ be a semihypergroup, i.e., $S$ is a nonempty set endowed with a hyperoperation $\circ: S \times$ $S \longrightarrow \mathcal{P}^{*}(S)$, so a function from the Cartesian product $S \times S$ to the family of nonempty subsets of $S$, which is associative: any three elements $a, b, c \in S$ satisfies the property $(a \circ b) \circ c=a \circ(b \circ c)$, where the left side member of the equality means the union of all hyperproducts $u \circ c$, with $u \in a \circ b$. A semihypergroup is a hypergroup if the reproduction axiom is verified, as well: for any $a \in S$, there is $a \circ S=S=S \circ a$. Sometimes, especially in the case of semihypergroups, when there is no risk of confusion, the hyperoperation is omitted, and the hyperproduct between two elements is simply denoted by $a b$, as we will do also throughout this paper. Besides, we denote the cardinality of a set $S$ by $|S|$. An element $a \in S$ is called left (right) scalar if $|a x|=1$ (respectively, $|x a|=1$ ), for all $x \in S$. If $a$ is both a left and right scalar, then it is called a two-sided scalar, or simply a scalar. An element $e$ in a semihypergroup $S$ is called the left (right) identity if $x \in e x$ (respectively, $x \in x e$ ), for all $x \in S$. If $e$ is both a left and an identity, then it is called a two-sided identity, or simply an identity. An element $e$ in a semihypergroup $S$ is called a left (right) scalar identity if $e x=\{x\}$ (respectively, $x e=\{x\}$ ), for all $x \in S$. If $e$ is both a left and right scalar identity, then it is called a two-sided scalar identity, or simply a scalar identity. An element $a^{\prime} \in S$ is called a left (right) inverse of the element $a \in S$ if there exists a left (right) identity $e \in S$ such that $e \in a^{\prime} a$ (respectively, $e \in a a^{\prime}$ ). If $a^{\prime}$ is both a left and right inverse of $a$, then it is called a two-sided inverse, or simply an inverse of $a$. An element zero in a semihypergroup $S$ is called a left (right) zero element if $0 x=\{0\}$ (respectively, $x 0=\{0\}$ ), for all $x \in S$. If zero is both a left and a right zero, then it is called a two-sided zero, or simply a zero.

A regular hypergroup is a hypergroup that has at least one identity and every element has at least one inverse. The regularity property for semihypergroups is not the same, in the sense that a semihypergroup $S$ is called regular if every element $x$ of $S$ is regular, i.e., if there exists $S \in S$ such that $x \in x_{S} x$. Notice that any regular semigroup is a regular semihypergroup, and moreover, based on the reproduction axiom, every hypergroup is a regular semihypergroup (but not necessarily a regular hypergroup).

Definition 1 ([12]). A polygroup is a system $\left\langle P, \circ, 1^{-1}\right\rangle$, where $1 \in P,-1$ is a unitary operation on $P, \circ$ maps $P \times P$ into the family of non-empty subsets of $P$, and the following axioms hold for all $x, y, z \in P$ :

$\left(P_{1}\right) x \circ(y \circ z)=(x \circ y) \circ z$,

$\left(P_{2}\right) 1 \circ x=x=x \circ 1$,

$\left(P_{3}\right) \quad x \in y \circ z$ implies $y \in x \circ z^{-1}$ and $z \in y^{-1} \circ x$.

Theorem 1. Let $S$ be a semihypergroup with the scalar identity element one such that every element $x \in S$ has a unique inverse, denoted by $x^{-1}$. Then, $S$ is a polygroup if and only if:

$\left(P_{3}^{\prime}\right) \forall x, y \in S,(x y)^{-1}=y^{-1} x^{-1}$

where $A^{-1}=\left\{a^{-1} \mid a \in A\right\}$ for $A \subseteq S$.

Proof. We can restrict our attention to the "only if" part, since the "if" part is obvious. Let $x, y, z \in S$ such that $x \in y z$. Then, $1 \in x x^{-1} \subseteq y\left(z x^{-1}\right)$, and thus, $y^{-1} \in z x^{-1}$. Hence, the property $\left(P_{3}^{\prime}\right)$ implies $y \in x z^{-1}$. Similarly, $z \in y^{-1} x$. Therefore, $S$ is a polygroup.

\section{Factorizable Semihypergroups}

Based on the definition of factorizable semigroups, in this section, we first introduce and study the main properties of factorizable semihypergroups. 
Definition 2. A semihypergroup $S$ is said to be factorizable if there exist some proper sub-semihypergroups $A$ and $B$ of $S$ such that $S=A B$ and $|a b|=1$ for every $a \in A$ and $b \in B$. The pair $(A, B)$ is called a factorization of $S$, with factors $A$ and $B$.

It is clear that every factorizable semigroup can be considered as a factorizable semihypergroup. In the following, we give some examples of factorizable proper semihypergroups (i.e., they are not semigroups).

Example 1. Let $S=\{1,2,3\}$ be a semihypergroup represented by the Cayley table:

\begin{tabular}{|c|ccc|}
\hline$\circ$ & 1 & 2 & 3 \\
\hline 1 & 1 & 1 & 1 \\
2 & 1 & 2 & 3 \\
3 & $\{1,2,3\}$ & 3 & $\{2,3\}$ \\
\hline
\end{tabular}

Then, the pair $(A, B)$, where $A=\{1,2\}$ and $B=\{2,3\}$, is a factorization of $S$.

Example 2. Let $S=\{1,2,3,4,5\}$ be a semihypergroup with the following Cayley table:

\begin{tabular}{|c|ccccc|}
\hline$\circ$ & 1 & 2 & 3 & 4 & 5 \\
\hline 1 & 1 & 2 & 3 & 4 & 5 \\
2 & 2 & 1 & 3 & 4 & 5 \\
3 & 3 & 3 & $\{1,2,3\}$ & 5 & $\{4,5\}$ \\
4 & 4 & 4 & 5 & $\{1,2,4\}$ & $\{3,5\}$ \\
5 & 5 & 5 & $\{4,5\}$ & $\{3,5\}$ & $\{1,2,3,4,5\}$ \\
\hline
\end{tabular}

Then, the pair $(A, B)$, where $A=\{1,2,3\}$ and $B=\{1,2,4\}$, is a factorization of $S$.

Lemma 1. If a semihypergroup $S$ is factorizable as $S=A B$, where $A$ has a left identity and $B$ has a right identity, then $S$ has a two-sided identity.

Proof. Suppose that $e$ is a left identity of the sub-semihypergroup $A$ and $e^{\prime}$ is a right identity of the sub-semihypergroup $B$. Since $e, e^{\prime} \in S$, we have $e=a b$ and $e^{\prime}=a^{\prime} b^{\prime}$ for some $a, a^{\prime} \in A$ and $b, b^{\prime} \in B$. Thus, $e=a b \in a\left(b e^{\prime}\right)=(a b) e^{\prime}=e e^{\prime}$; thus, $e=e e^{\prime}$. Similarly, one obtains $e^{\prime}=e e^{\prime}$. Therefore, $e=e^{\prime} \in A \cap B$ is a two-sided identity element of $S$.

Similarly, we can prove the following lemma.

Lemma 2. If a semihypergroup $S$ is factorizable as $S=A B$, where $A$ has a left scalar identity and $B$ has a right scalar identity, then $A, B$, and $S$ have a two-sided scalar identity.

In the next theorem, we present a sufficient condition such that a factorizable semihypergroup is a hypergroup.

Theorem 2. If a semihypergroup $S$ is factorizable as $S=A B$, where $A$ and $B$ are hypergroups such that $A \cap B \neq \varnothing$, then $S$ is a hypergroup.

Proof. Let $S$ be a semihypergroup and $(A, B)$ a factorization of $S$, where $A$ and $B$ are hypergroups such that $e \in A \cap B$. Then, for every $x \in S$, there exist $a \in A$ and $b \in B$ such that $x=a b$. By the reproducibility property of $A$ and $B$, there exist $a^{\prime}, a^{\prime \prime}, a^{*} \in A$ and $b^{\prime}, b^{\prime \prime}, b^{*} \in B$ such that $a \in a^{\prime} a^{\prime \prime}$, $a^{\prime \prime} \in e a^{*}, b \in b^{\prime} b^{\prime \prime}$, and $e \in b^{\prime} b^{*}$. Thus, we can write:

$$
x=a b \in\left(a^{\prime} a^{\prime \prime}\right) b \subseteq a^{\prime}\left(e a^{*}\right) b \subseteq a^{\prime}\left(b^{\prime} b^{*}\right) a^{*} b=\left(a^{\prime} b^{\prime}\right)\left(b^{*} a^{*} b\right) .
$$


Hence, $x \in\left(a^{\prime} b^{\prime}\right) z=y z$, with $z \in b^{*} a^{*} b$ and $y=a^{\prime} b^{\prime}$. Similarly, one proves that $x \in t y$, with $t \in S$, meaning that the reproducibility property in $S$ holds, as well. Therefore, $S$ is a hypergroup.

The following example shows that the converse of the above theorem does not hold necessarily.

Example 3. Let $S=\{1,2,3,4,5,6\}$ be a hypergroup with the Cayley table:

\begin{tabular}{|c|cccccc|}
\hline$\circ$ & 1 & 2 & 3 & 4 & 5 & 6 \\
\hline 1 & 1 & 2 & 3 & 4 & 5 & 6 \\
2 & 2 & 1 & 4 & 3 & 6 & 5 \\
3 & 1 & 2 & 3 & 4 & 5 & 6 \\
4 & 2 & 1 & 4 & 3 & 6 & 5 \\
5 & $\{1,3,5\}$ & $\{2,4,6\}$ & $\{1,3,5\}$ & $\{2,4,6\}$ & $\{1,3,5\}$ & $\{2,4,6\}$ \\
6 & $\{2,4,6\}$ & $\{1,3,5\}$ & $\{2,4,6\}$ & $\{1,2,5\}$ & $\{2,4,6\}$ & $\{1,3,5\}$ \\
\hline
\end{tabular}

Then, the pair $(A, B)$, where $A=\{1,2,3,4\}$ and $B=\{1,3,5\}$, is a factorization of $S$, but $A$ is not $a$ hypergroup, while $B$ is a hypergroup.

Theorem 3. Let the semihypergroup $S$ be factorizable as $S=A B$, where $A$ is a group. If $B$ is contained in the semihypergroup class $C_{i},(i=1,2,3)$, then so, $S$ is, where:

$C_{1}=$ the class of regular semihypergroups;

$C_{2}=$ the class of hypergroups;

$C_{3}=$ the class of regular hypergroups.

Proof. We denote by $e$ the identity of the group $A$, which is, based on Lemma 1, a left scalar identity of $S$.

(1) Assume that $B$ is a regular semihypergroup, and let $x=a b$ be an arbitrary element in $S=A B$. Then, there exits $b^{\prime} \in B$ such that $b \in b b^{\prime} b$. Thus, $x=a b \in a b b^{\prime} a^{-1} a b$ and, thereby, $x \in x x^{\prime} x$, for some $x^{\prime} \in b^{\prime} a^{-1}$. This means that any element in $S$ is regular, so the semihypergroup $S$ is regular, as well.

(2) Assume that $B$ is a hypergroup and $S=A B$. Then there exist $a \in A$ and $b \in B$ such that $e=a b$. Thus $a^{-1}=e b=b \in A \cap B$. So, by Theorem 2, we conclude that $S$ is a hypergroup.

(3) Assume that $B$ is a regular hypergroup. Then, by Lemma 1, the identity elements of $S, A$, and $B$ are the same. Let $x=a b$, with $a \in A$ and $b \in B$, be an arbitrary element of $S$ and $y \in b^{-1} a^{-1}$. Then, $y a=b^{-1}$; hence, $1 \in b^{-1} b=y a b$, so $y=(a b)^{-1}$. Thus, $(a b)^{-1}=b^{-1} a^{-1}$. Therefore, $S$ is a regular hypergroup.

Definition 3. A factorization ( $A, B)$ of a semihypergroup $S$ is called left univocal (respectively right univocal) if the following condition holds:

$$
\left(\forall a, a^{\prime} \in A, b, b^{\prime} \in B\right) a b=a^{\prime} b^{\prime} \Longrightarrow a=a^{\prime} \quad\left(\text { respectively, } b=b^{\prime}\right) .
$$

A factorization $(A, B)$ of a semihypergroup $S$ is called univocal if it is both left and right univocal.

Example 4. Let $S=\{1,2,3,4\}$ be a semihypergroup with the following Cayley table:

\begin{tabular}{|c|cccc|}
\hline$\circ$ & 1 & 2 & 3 & 4 \\
\hline 1 & 1 & 2 & 3 & 4 \\
2 & 2 & $\{1,2\}$ & 4 & $\{3,4\}$ \\
3 & 3 & 4 & $\{1,3\}$ & $\{2,4\}$ \\
4 & 4 & $\{3,4\}$ & $\{2,4\}$ & $\{1,2,3,4\}$ \\
\hline
\end{tabular}


Then, the pair $(A, B)$, where $A=\{1,2\}$ and $B=\{1,3\}$, is a left univocal factorization of $S$.

Example 5. Let $S=\{1,2,3,4\}$ be a semihypergroup with the following Cayley table:

\begin{tabular}{|c|cccccc|}
\hline$\circ$ & 1 & 2 & 3 & 4 & 5 & 6 \\
\hline 1 & 1 & 2 & 3 & 4 & 5 & 6 \\
2 & 2 & 1 & 3 & 5 & 4 & 6 \\
3 & 3 & 3 & $\{1,2\}$ & 6 & 6 & $\{4,5\}$ \\
4 & 4 & 5 & 6 & 1 & 2 & 3 \\
5 & 5 & 4 & 6 & 2 & 1 & 3 \\
6 & 6 & 6 & $\{4,5\}$ & 3 & 3 & $\{1,2\}$ \\
\hline
\end{tabular}

Then, the pair $(A, B)$, where $A=\{1,4\}$ and $B=\{1,2,3\}$, is a left univocal factorization of $S$.

Lemma 3. Let $(A, B)$ be a left univocal factorization of a semihypergroup $S$. Then, the following assertions hold:

(i) If $a=a^{\prime} b$ for $a, a^{\prime} \in A$ and $b \in B$, then $a=a^{\prime}$.

(ii) Let $a \in A, A_{1} \subseteq A$, and $B_{1} \subseteq B$ be such that $a \in A_{1} B_{1}$. Then, $a \in A_{1}$.

(iii) Let $a \in A, A_{1} \subseteq A, b \in B$, and $B_{1} \subseteq B$ be such that $a b \in A_{1} B_{1}$. Then, $a \in A_{1}$.

(iv) If $a \in A_{1} b$, with $A_{1} \subseteq A$, then $a \in A_{1}$ and $a=a b$.

(v) For every $a \in A$, there exists $b \in B$ such that $a=a b$.

\section{Proof.}

(i) Suppose that $a=a^{\prime} b$ for $a, a^{\prime} \in A$ and $b \in B$. Then, $a b \in a^{\prime}(b b)$, and thus, $a b=a^{\prime} b^{\prime}$ for $b^{\prime} \in b b$, which implies that $a=a^{\prime}$.

(ii) Let $a \in A, A_{1} \subseteq A$ and $B_{1} \subseteq B$ be such that $a \in A_{1} B_{1}$. Then, we have $a=a_{1} b_{1}$, with $a_{1} \in A_{1}$ and $b_{1} \in B_{1}$. Hence, Assertion (i) implies that $a=a_{1} \in A_{1}$.

(iii) Let $a \in A, A_{1} \subseteq A, b \in B$, and $B_{1} \subseteq B$ be such that $a b \in A_{1} B_{1}$. Then, we have $a b=a_{1} b_{1}$, with $a_{1} \in A_{1}$ and $b_{1} \in B$, so $a=a_{1} \in A_{1}$.

(iv) This follows immediately from Assertion (i).

(v) For every $a \in A$, there exist $a^{\prime} \in A$ and $b \in B$ such that $a=a^{\prime} b$. Then, Assertion (i) implies $a=a^{\prime}$, as required.

Lemma 4. Let $(A, B)$ be a left univocal factorization of a semihypergroup $S$. Then, $A \cap B$ is a left zero semigroup formed by right scalar identities of $A$.

Proof. First we prove that the intersection $A \cap B$ is nonempty. Let $b$ be an element of $B$ such that $b=e b^{\prime}$, with $e \in A$ and $b^{\prime} \in B$. Then, for every $a \in A$, it follows that $a b \in(a e) b^{\prime}$; thus, Lemma 3 (iii) implies $a \in a e$. Again, by Lemma $3(\mathrm{v})$, there exists $b^{\prime \prime} \in B$ such that $e=e b^{\prime \prime}$, while $b^{\prime \prime}=a^{*} b^{*}$, with $a^{*} \in A$ and $b^{*} \in B$. Thus, $a^{*} b^{\prime \prime} \in\left(a^{*} a^{*}\right) b^{*}$. It follows that $a^{*} \in a^{*} a^{*}$ by Lemma 3 (iii). Hence, $a^{*} \in a^{*} e=a^{*} e b^{\prime \prime}$, so $a^{*}=a^{*} b^{\prime \prime}$ by Lemma 3 (iv). Thereby, $a^{*}=a^{*} b^{\prime \prime}=\left(a^{*} a^{*}\right) b^{*}$, and thus, $a^{*}=a^{*} b^{*}=b^{\prime \prime}$, which shows that $A \cap B \neq \varnothing$.

Now, let $e$ be an arbitrary element in $A \cap B$. Then, ee $\in A \cap B$, with $|e e|=1$ and $e(e e)=(e e) e$; thus, $e=e e$, because of the univocal factorization. Moreover, since $a e=(a e) e$, it follows that $a=a e$; thus, $e$ is a scalar right identity of $A$. Therefore, $A \cap B$ is a left zero sub-semigroup of $S$ formed by right scalar identities of $A$.

Remark 1. Note that the property "to be a scalar" is essential in Lemma 4, i.e., if $(A, B)$ is a left univocal factorization of a semihypergroup $S$ and e is a right identity, but not a scalar one, then $e \notin A \cap B$, as we can see in the following example. 
Example 6. Let $S=\{1,2,3,4\}$ be a semihypergroup with the following Cayley table:

\begin{tabular}{|c|cccc|}
\hline$\circ$ & 1 & 2 & 3 & 4 \\
\hline 1 & 1 & $\{1,2\}$ & 1 & 1 \\
2 & 1 & $\{1,2\}$ & 1 & 1 \\
3 & 1 & 2 & $\{3,4\}$ & 3 \\
4 & 1 & 2 & $\{3,4\}$ & 4 \\
\hline
\end{tabular}

Then, the pair $(A, B)$, where $A=\{3,4\}$ and $B=\{1,2,4\}$, is a factorization of $S$. We observe that the element 3 is a right, but not scalar, identity of $A$, which does not belong to $A \cap B$.

Theorem 4. Let $(A, B)$ be a left univocal factorization of a polygroup $P$. Then, the following assertions hold:

(i) $A$ and $B$ are sub-polygroups of $P$.

(ii) $A \cap B=\{1\}$, where one is the identity of the polygroup $P$.

\section{Proof.}

(i) Let $P$ be a polygroup with the identity one and $(A, B)$ be a left univocal factorization of $P$. First, we prove that both $A$ and $B$ contain one. Since there exists $a \in A$ such that $1=a a^{-1}$, it follows that $a \in a^{2} a^{-1}$, and hence, $a=a^{\prime} a^{-1}$ for some $a^{\prime} \in a^{2}$. Thus, $a \in a a^{-1}=1$; hence, $1=a=a^{-1} \in A \cap B$.

Now, for every $a \in A$, there exist $a^{\prime} \in A$ and $b^{\prime} \in B$ such that $a^{-1}=a^{\prime} b^{\prime}$. Thus, $1 \in a a^{-1}=a a^{\prime} b^{\prime}$; and hence, $1 \in a a^{\prime}$, by Lemma 3 (ii). This means that $A$ is a polygroup.

Furthermore, let $b \in B$. Then, $b^{-1}=a^{\prime} b^{\prime}$ with $a^{\prime} \in A$ and $b^{\prime} \in B$. Thus, $1 \in b^{-1} b=a^{\prime} b^{\prime} b$, so $a^{\prime}=1$, by Lemma 3 (iv). Hence, $b^{-1}=b^{\prime} \in B$, meaning that $B$ is a polygroup.

(ii) This follows from Assertion (i) and Lemma 4.

Example 7. Let $P=\{1,2,3,4,5,6\}$ be a polygroup with the following Cayley table:

\begin{tabular}{|c|cccccc|}
\hline$\circ$ & 1 & 2 & 3 & 4 & 5 & 6 \\
\hline 1 & 1 & 2 & 3 & 4 & 5 & 6 \\
2 & 2 & 1 & 3 & 5 & 4 & 6 \\
3 & 3 & 3 & $\{1,2\}$ & 6 & 6 & $\{4,5\}$ \\
4 & 4 & 5 & 6 & 1 & 2 & 3 \\
5 & 5 & 4 & 6 & 2 & 1 & 3 \\
6 & 6 & 6 & $\{4,5\}$ & 3 & 3 & $\{1,2\}$ \\
\hline
\end{tabular}

Then, the pair $(A, B)$ where $A=\{1,2,3\}$ and $B=\{1,4\}$ is a factorization of $P$.

Lemma 5. Let $(A, B)$ be a left univocal factorization of a polygroup $P$. Then, $|P|=|A| \cdot|B|$.

Proof. Let $(A, B)$ be a left univocal factorization of a polygroup $P$. Since $P=A B$, it is sufficient to prove that for every $x \in P$, there exist unique $a \in A$ and $b \in B$ such that $x=a b$. If $x=a b=a^{\prime} b^{\prime}$, then, by definition, we get $a=a^{\prime}$, and hence, $a b=a b^{\prime}$. Therefore, $b \in a a^{-1} b^{\prime}$. Thus, there exists $a^{\prime \prime} \in A$ such that $b \in a^{\prime \prime} b^{\prime}$. Hence, $a^{\prime \prime} \in b b^{\prime-1}$. It follows that $a^{\prime \prime} \in A \cap B$, meaning that $a^{\prime \prime}=1$, by Theorem 4 . We conclude that $b=b^{\prime}$.

Proposition 1. The minimum order of a non-commutative left univocal factorizable polygroup is six.

Proof. First, we show that every left univocal factorizable polygroup of order less than six is commutative. Let $(A, B)$ be a left univocal factorization of a polygroup $P$ and $|P|<6$. Then, Lemma 5 
implies $|P|=4$ and $|A|=|B|=2$. Consider $P=\{1, a, b, c\}, A=\{1, a\}$, and $B=\{1, b\}$. Since $A$ and $B$ are sub-polygroups of $P$, by Theorem 2, we have $a=a^{-1}$ and $b=b^{-1}$, and hence, $c=c^{-1}$. Now, for every $x, y \in P$ we have:

$$
x y=(x y)^{-1}=y^{-1} x^{-1}=y x .
$$

Therefore, $P$ is a commutative polygroup. Now, one can see that the symmetric group on the

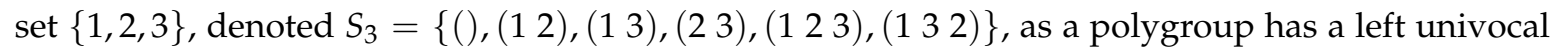
factorization $S_{3}=\left\{(),\left(\begin{array}{ll}1 & 2\end{array}\right)\right\}\left\{(),\left(\begin{array}{lll}1 & 2 & 3\end{array}\right),\left(\begin{array}{lll}1 & 3 & 2\end{array}\right)\right\}$, which completes the proof.

\section{Conclusions}

Many properties from semigroup theory have been extended to semihypergroup theory, showing their similarities but also differences, and the factorization property, discussed within this note, is one of them. Following the classical paper of Catino [1], we defined a factorizable semihypergroup as a semihypergroup that can be written as a hyperproduct of its two proper sub-semihypergroups. One of the main results presented here showed that if the semihypergroup $S$ is factorized as $S=A B$ with $A$ a group and $B$ a regular semihypergroup, a hypergroup, or a regular hypergroup, then also $S$ has the same algebraic hypercompositional structure as $B$. Regarding the polygroups, it was proven that for a left univocal factorizable polygroup $P=A B$, both factors are sub-polygroups of $P$, with the intersection containing only the identity of $P$. Moreover, we determined that the minimum order of a non-commutative left univocal factorizable polygroup is six.

Author Contributions: Conceptualization, D.H. and I.C.; funding acquisition, I.C.; investigation, D.H. and I.C.; methodology, D.H. and I.C.; writing, original draft, D.H.; writing, review and editing, I.C. All authors read and agreed to the published version of the manuscript.

Funding: The second author acknowledges the financial support from the Slovenian Research Agency (Research Core Funding No. P1-0285).

Conflicts of Interest: The authors declare no conflict of interest.

\section{References}

1. Catino, F. Factorizable semigroups. Semigroup Forum 1987, 36, 167-174. [CrossRef]

2. Tolo, K. Factorizable semigroups. Pac. J. Math. 1969, 31, 523-535. [CrossRef]

3. Amberg, B.; Kazarin, L.S. Factorizations of groups and related topics. Sci. China Ser. A Math. 2009, 52, $217-230$. [CrossRef]

4. Redei, L. Algebra I; Pergamon Press: Oxford, UK, 1967.

5. Heidari, D.; Cristea, I. Breakable semihypergroups. Symmetry 2019, 11, 100. [CrossRef]

6. Ito, N. Uber das Produkt von zwei abelschen Gruppen. Math. Z. 1955, 62, 400-401. [CrossRef]

7. Tirasupa, Y. Factorizable transformation semigroups. Semigroup Forum 1979, 18, 15-19. [CrossRef]

8. Massouros, C.G. Separation and Relevant Properties in Hypergroups. AIP Conf. Proc. 2016, 1738, 480051-1-480051-4.

9. Massouros, C.G. On connections between vector spaces and hypercompositional structures. Int. J. Pure Appl. Math. 2015, 34, 133-150.

10. Corsini, P. Prolegomena of Hypergroup Theory; Aviani Editore: Tricesimo, Italy, 1993.

11. Davvaz, B. Semihypergroup Theory; Elsevier/Academic Press: London, UK, 2016.

12. Davvaz, B. Polygroup Theory and Related Systems; World Sciences Publishing Co. Pte. Ltd.: Hackensack, NJ, USA, 2013.

13. Heidari, D.; Davvaz, B. Characterization of small polygroups by their fundamental groups. Discret. Math. Algorithms Appl. 2019, 11, 1950047. [CrossRef]

14. Heidari, D.; Amooshahi, M.; Davvaz, B. Generalized Cayley graphs over polygroups. Commun. Algebra 2019, 47, 2209-2219. [CrossRef]

(C) 2020 by the authors. Licensee MDPI, Basel, Switzerland. This article is an open access article distributed under the terms and conditions of the Creative Commons Attribution (CC BY) license (http://creativecommons.org/licenses/by/4.0/). 